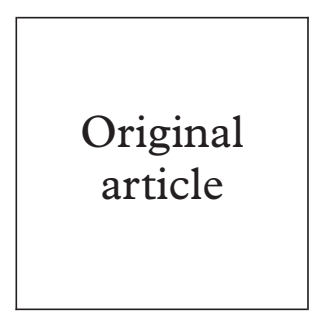

\title{
Sexually transmitted infections and HIV in a rural community in the Lesotho highlands
}

\author{
M Colvin, B Sharp
}

Objectives: To conduct a knowledge, attitude, and practice (KAP) study and to determine the prevalence of sexually transmitted diseases (STDs), including HIV, in a community residing in remote, rural Lesotho.

Methods: In 1995 a cross sectional, community based epidemiological study was conducted on a population of 7500 people living in 89 villages. A total of 29 villages were randomly selected and a systematic sample of houses within villages was obtained. Questionnaires were administered to subjects after written consent was obtained. Determination of $N$ gonococcus and $C$ trachomatis infection was done on urine using ligase chain reaction (LCR) technology. Using blood specimens, syphilis was diagnosed by RPR and TPHA tests and HIV by a single ELISA and confirmed with a western blot.

Results: Questionnaires were administered to 277 women, 100 men, and 149 youths (12-15 years). Chlamydia was diagnosed in $28.4 \%$ of adults, gonorrhoea in $5.9 \%$, syphilis in $11.3 \%$, and HIV infection in $6.3 \%$. All cases of HIV occurred along the main road $(p=0.001)$ and $72 \%$ of individuals with gonorrhoea were co-infected with chlamydia $(p=0.0001) .11 .6 \%$ of women and $38.0 \%$ of men had had sex with a non-regular partner in the past 3 months and none had used condoms.

Conclusion: A high prevalence of STDs and HIV infection was found in a population characterised by low levels of knowledge about STD/HIV, high risk sexual behaviour, and evidence of inappropriate health seeking behaviour for STDs.

(Sex Transm Inf 2000;76:39-42)

Keywords: HIV; sexually transmitted infections

\section{Introduction}

At present, the most severe HIV epidemics in the world are occurring in southern African countries with prevalence among antenatal clinic attenders being over $40 \%$ in certain urban areas. ${ }^{1}$ It is believed that the epidemic in the region is promoted by multiple factors including the high prevalence of sexually transmitted diseases (STDs), ${ }^{2}$ a lack of access to effective STD treatment, the prevalent sexual networking patterns, and the ongoing disruptive effects on the subcontinent of the apartheid created migrant labour system. ${ }^{3-5}$

There have been no published reports on the prevalence of STDs in the landlocked southern African kingdom of Lesotho and only one study on HIV levels. ${ }^{6}$ The latter study, conducted in 1992, revealed a low prevalence of HIV among mountain villagers $(0.8 \%)$ but a relatively high prevalence of $5.3 \%$ among the Katse dam's labour force. Concerns that this massive dam and tunnel construction project, currently under way in the mountains, may promote the transmission of these infections through improved transport links and the influx of a labour force, were the main motivating factors for this study. The purpose was, therefore, to determine the baseline prevalence of STDs and HIV among the rural dwellers in the phase $1 \mathrm{~B}$ area before any development began on the Mohale dam and to propose measures to mitigate against and monitor the spread of STDs and HIV

\section{Methods}

A cross sectional study was conducted during 1995 in the Mohale catchment area (fig 1) which comprises 400 square kilometres of remote, mountainous territory about $70 \mathrm{~km}$ east of the capital, Maseru. The study population, estimated to be at least 7500 people, live in 83 scattered villages. A random sample, stratified on distance from the proposed development, of 29 villages, was selected for inclusion into the study. All adults between the ages of 15 and 49 years of age were eligible for inclusion in the study.

Informed, written consent was obtained from each subject and a structured questionnaire, translated and back translated into the local vernacular, was then administered to elicit information on household demographics, sexual knowledge and behaviour, and the occurrence of sexually transmitted infections.

"First catch" urine specimens were taken, stored at about $4^{\circ} \mathrm{C}$ and transported to the field laboratory within 24 hours where they were frozen to $-22^{\circ} \mathrm{C}$. Each week the specimens were taken to the main laboratory in Maseru where they were stored at $-70^{\circ} \mathrm{C}$ before being transported to the SAIMR laboratories in Johannesburg, South Africa, for determination of Neisseria gonococcus and Chlamydia trachomatis infection using ligase chain reaction (LCR) technology (Abbott, Chicago, IL, USA).

Venous blood was obtained and stored at about $4^{\circ} \mathrm{C}$ before being transported daily to the field laboratory. After processing the serum was 


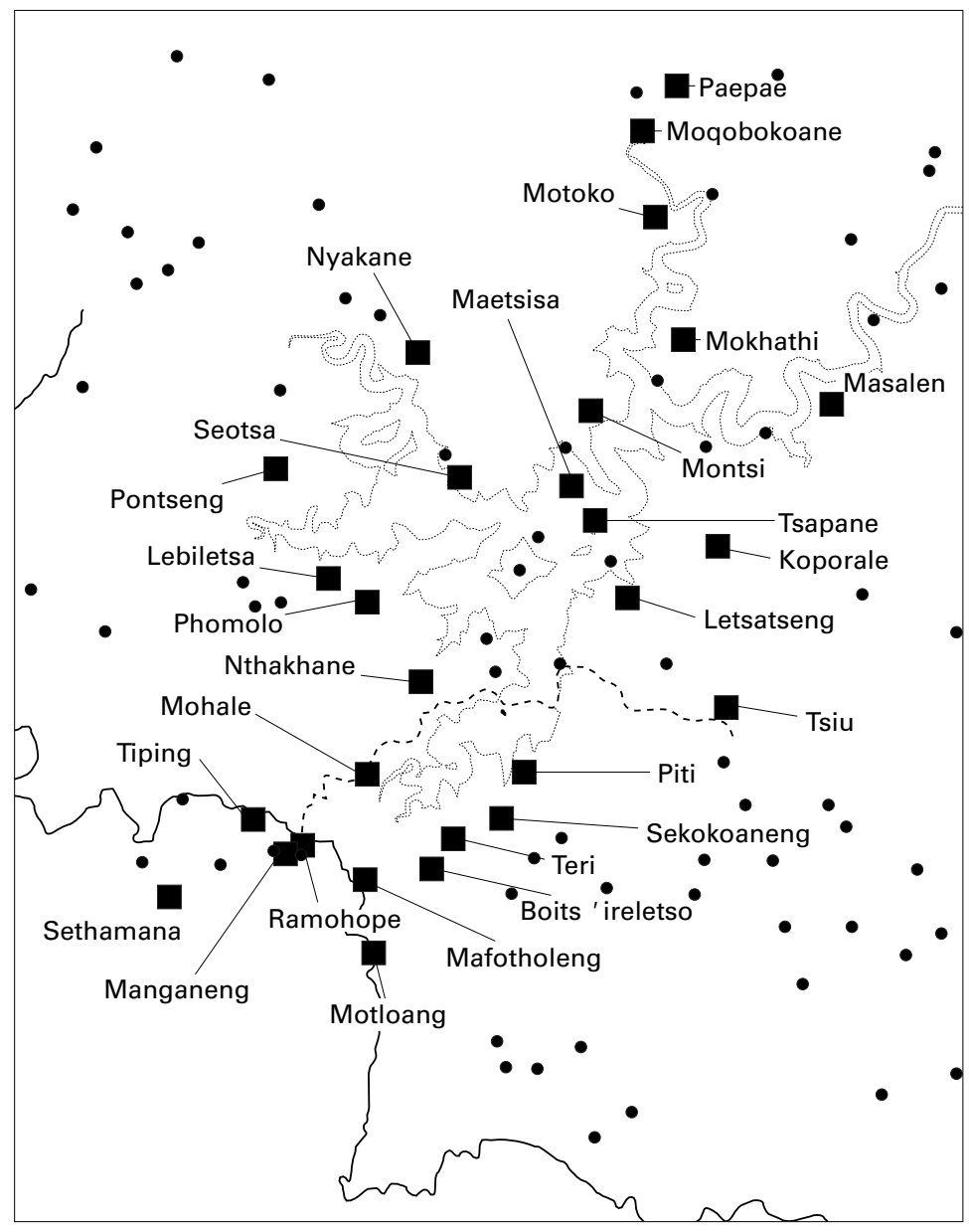

Figure 1 A map of the phase $1 B$ area showing the 29 sampled villages in relation to the main road and to where flooding will occur when the Mohale dam is completed.

stored at $-22^{\circ} \mathrm{C}$ and twice weekly samples were taken to Maseru, stored at $-70^{\circ} \mathrm{C}$, and forwarded to the SAIMR laboratories for determination of syphilis and HIV infection. Syphilis serology comprised the rapid plasma reagin (RPR) test (Brewer Diagnostic Kits, BBL Microbiology Systems, MD USA) and the Treponema pallidum haemagglutination assay (TPHA) (Immutrep TPHA, Omega Diagnostics Ltd, UK). A reactive test for both RPR and TPHA was regarded as an indicator of active syphilis. A positive TPHA result combined with a negative RPR result was considered to indicate a history of successfully treated syphilis in the past. HIV serology was done unlinked and comprised a recombinant HIV1/ HIV2 ELISA test (Abbott Laboratories). All reactive ELISA tests were confirmed by western blot (Diagnostic Biotechnology, Singapore).
All subjects who were diagnosed as having a non-viral STD were individually notified and arrangements made for them to receive appropriate treatment at the nearest health post. The protocol for this study was approved by the ethics committee of the Medical Research Council of South Africa, the Lesotho Highlands Development Authority, and the Lesotho Ministry of Health.

\section{Results}

A total of 277 adult women, 100 men, and 149 youths (12-15 years) were given questionnaires. Formal response rates could not be calculated as it was difficult to differentiate absentees from refusals. However, it was estimated that about $25 \%$ of homes approached refused to participate. Of the 377 adults who were given questionnaires, urine samples were obtained from $306(81.2 \%)$ individuals and blood samples were obtained from 238 (63\%) individuals.

The mean age of the women was 33.0 (SD 8.7) years and men 29.9 (8.4) years. The mean number of years of schooling for women was 6.1 (2.17) years and for men only 2.4 (2.70) years.

The prevalence of sexually transmitted infections among adults is given in table 1 . All cases of HIV infection occurred among those subjects living along the main road (15 of 115) and no cases were detected among the 123 subjects living off the main road $(p=0.001)$. There were more cases of gonococcal infection and HIV among women but these did not reach statistical significance. The prevalence of chlamydia co-infection in individuals with gonorrhoea was $72 \%$ whereas there was a $26 \%$ prevalence of chlamydia infection in people without gonorrhoea $(\mathrm{p}=0.00002)$.

Over the past year, 80 women $(29 \% ; 95 \%$ CI 23.6-34.6) had experienced a vaginal discharge, 50 (18\%; 95\% CI 13.7-23.1) had noticed pain on micturition, and eight $(3 \%$; 95\% CI 1.3-5.6) complained of "sores in the anogenital area." Medical treatment was sought by $114(41 \% ; 95 \%$ CI $35.3-47.2)$ of those who had symptoms; $58(21 \% ; 95 \%$ CI 16.3-26.2) used herbs or consulted a traditional healer and 91 (33\%; 95\% CI 27.438.7) sought no treatment. Lack of money and mild symptoms were the main reason for not seeking treatment. During the same time period, 11 (11\%; 95\% CI 5.6-18.8) men experienced a penile discharge, five $(5 \% ; 1.6-11.3)$ had sores in the anogenital area, and eight $(8 \%$; 95\% CI 3.5-15.1) complained of dysuria. Men had very similar health seeking behaviour patterns to the women.

Table 1 Prevalence of STDs by sex

\begin{tabular}{|c|c|c|c|c|c|c|c|c|}
\hline \multirow[b]{2}{*}{$S T D$} & \multicolumn{4}{|l|}{ Women } & \multicolumn{4}{|l|}{ Men } \\
\hline & Sample & No infected & $\%$ & $95 \% C I$ & Sample & No infected & $\%$ & $95 \% C I$ \\
\hline Chlamydia trachomatis & 245 & 71 & 29 & $23.3-34.7$ & 59 & 16 & 27.1 & $15.8-38.5$ \\
\hline Gonorrhoea & 245 & 17 & 6.9 & $3.8-10.1$ & 59 & 1 & 1.7 & $0.0-4.9$ \\
\hline RPR and TPHA+ve & 190 & 22 & 11.6 & $7.0-16.1$ & 45 & 5 & 10.9 & $1.9-19.8$ \\
\hline RPR-ve, TPHA+ve & 190 & 42 & 22.2 & $16.0-28.3$ & 45 & 8 & 17.8 & $6.6-28.9$ \\
\hline HIV & 197 & 14 & 7.1 & $3.5-10.7$ & 45 & 1 & 2.4 & $0.0-7.2$ \\
\hline
\end{tabular}


Fertility rates were high with 275 (99\%; $95 \%$ CI 97.4-99.9) women respondents having been pregnant at some time in their lives. By the age of 20 years $206(75 \% ; 68.8-79.4)$ women had been pregnant at least once. Although 230 (83\%; 95\% CI 78.1-87.3) women who had been pregnant attended antenatal care, 154 (56\%; 95\% CI 49.5-61.5) gave birth at home. Lifetime use of any contraceptive was limited to $75(27 \% ; 95 \%$ CI $21.9-32.7)$ women with $46(17 \% ; 95 \%$ CI 12.4-21.5) having used injectables, $33(12 \%$; $95 \%$ CI $8.3-16.3)$ oral contraceptives, and only nine $(3 \% ; 95 \%$ CI $1.5-6.1)$ had ever used condoms.

Mens' attitudes towards contraception were not favourable, with 43 (43\%; 95\% CI $33.1-$ 53.3) agreeing with the statement that "contraception is against God's will" and $61(61 \% ; 95 \%$ CI 50.7-70.6) agreeing that it is sometimes too embarrassing to ask for contraceptives. Interestingly, more women $(244,88 \% ; 95 \%$ CI $83.3-91.3)$ than men (65, 65\%; 95\% CI 54.8-74.3) agreed with the statement that "husbands may not approve of contraceptives."

While the majority of women (253, 91\%; 95\% CI 87.4-94.3) had heard about AIDS and 236 (85\%; 95\% CI 80.5-89.2) knew that condoms afforded protection against infection, misconceptions were common with 134 (48\%; 95\% CI 42.4-54.4) thinking that AIDS could be transmitted through sharing eating utensils. There was also a discrepancy between knowledge and behaviour as demonstrated by the fact that none of the $32(12 \% ; 95 \%$ CI 8.0-15.9) women who admitted having had sex with a non-regular partner in the past 3 months had used condoms.

Men had less knowledge of AIDS than women with only $80(80 \%$; 95\% CI 70.1-87.3) having heard about it and, of this group, only 53 $(53 \% ; 95 \%$ CI $42.8-63.1)$ were sure that one could protect oneself from HIV infection. The myth that having sex with a virgin could cure AIDS is prevalent with 25 (25\%; 95\% CI 17.0 34.6) men believing this. Only 17 (17\%; $95 \%$ CI 10.2-25.8) men had ever used condoms and none of the $38(38 \% ; 28.5-48.2)$ who had sex with a non-regular partner in the past 3 months used condoms during this period.

The main source of information with regard to AIDS was the radio ( 78 women, $28 \% ; 95 \%$ CI 22.9-33.9, and 55 men, 55\%; 95\% CI 44.7-64.9) followed by the clinic (58 women, $21 \% 95 \%$ CI 16.3-26.2, and five men, 95\% CI $5 \% 1.6-11.2$ ) and other villagers (44 women, $16 \%, 95 \%$ CI $11.8-20.7$, and 25 men, $25 \%$ 95\% CI 16.9-34.6).

Of the youths interviewed, the majority (85, $57 \%$; 95\% CI 48.7-65.1) were scholars, with 22 (15\%; 95\% CI 9.5-21.5) engaged in housework and 37 (25\%; 95\% CI 18.1-32.5) being herders. Many more girls (64, 75\%; 95\% CI $63.9-83.2)$ than boys $(18,29 \%$; $95 \%$ CI 17.9 41.3 ) attended school mainly because 41 boys (65\%; 95\% CI 52.0-76.6) were herders. Only 99 (66\%; 95\% CI 58.2-73.9) youths claimed to have heard about AIDS and more girls (68; $79 \%$; 95\% CI 68.9-87.1) than boys (30; 48\%; 95\% CI 34.9-60.6) had heard about it $(\mathrm{p}<0.0001)$. Of this group, only $30(30 \% ; 95 \%$ CI 21.4-40.3) were sure that there was a way of protecting oneself from AIDS. Information on sexual matters was overwhelmingly obtained from friends $(94,63 \%$; 95\% CI 54.8-70.8) or school $(34,23 \% ; 16.3-30.4)$ with little information being obtained from parents (eight, $5 \%$; $95 \%$ CI $2.3-10.3$ ) or the radio (six, $4 \%$; 95\% CI 1.5-8.6). Knowledge about STDs was limited with only 33 (22\%; 95\% CI 15.8-29.7) having heard of them.

\section{Discussion}

This study has demonstrated a high prevalence of STDs and HIV infection in a population characterised by low levels of knowledge about STD/HIV, high risk sexual behaviour (low condom use and multiple partners) and evidence of inappropriate health seeking behaviour for STDs. The situation is likely to be exacerbated in this particular area by the imminent influx of several thousand male workers, most of whom will come without families. All these factors will promote the spread of STDs, including HIV, unless drastic steps are taken to contain the epidemics.

The finding that all cases of HIV occurred in villages along the only main road in the area, supports the theory that HIV transmission enters an area along arterial transport routes. ${ }^{7}$ The Nairobi-Mombasa highway has been associated with HIV infection ${ }^{89}$ as has the Trans-African highway in East Africa. ${ }^{10}$ In Mwanza, Tanzania, the prevalence of HIV was twice as high in communities living along the roadside in comparison with those living in villages distant to the main road. ${ }^{11}$ As dam construction starts and traffic flow increases on the improved roads it may be anticipated that this development will also boost increased STD and HIV transmission along these routes with subsequent spread into adjoining areas.

The high rates of chlamydia co-infection in subjects with gonorrhoea highlight the need for syndromic management of STDs. However, syndromic management only impacts on those people who present to the health services and there is evidence from this study that less than half the men with symptoms of an STD will consult a doctor. Clearly there is a need in this community to improve both health seeking behaviours and knowledge about STDs and HIV.

The high proportion of men and women who reported having had recent sex with a non-regular partner supports previously reported data which found that men and women in Lesotho had the highest rate of current multiple partnerships of 11 surveyed developing countries. ${ }^{12}$ The fact that nobody in this study had used condoms with non-regular partners puts this community at high risk for the transmission of HIV and other STDs.

As the majority of adults obtain their information from the radio or clinics it would be appropriate to target the adult population through these channels. The youths, on the other hand, overwhelmingly obtain their information from peers and the school and so for 
this group peer education schemes and school based campaigns need to be developed.

Communities in this area are concerned about the impending loss of their homes, land, and livelihood to the dam construction programme and part of their response is to not cooperate with the myriad of baseline studies currently being conducted in the area. Of concern in this study is whether or not non-response is related the presence of an STD or to people's sexual practices and hence whether a bias may have been introduced. As this was a baseline health study, of which sexual infections and practices were a small component, it is unlikely that non-response was specifically related to an individuals risk of having an STD or to any particular sexual practices. Because of this we argue that the sample is unlikely to be biased.

In conclusion, this study has demonstrated the heavy burden of disease due to STDs in the study area and highlighted the lack of knowledge and capacity of this poor rural community to protect themselves from becoming infected. The researchers made specific proposals with regard to the provision of health services and health education and recommended a monitoring and audit programme. The health authorities need to act decisively in order to control these epidemics and prevent them becoming exacerbated by the construction programme.

1 UNAIDS and WHO. Report on the global HIV/AIDS epidemic-fune 1998. Geneva: WHO, 1998.

2 Pham-Kanter GBT, Steinberg MH, Ballard RC. Sexually transmitted diseases in South Africa.. Genitourin Med 1996;72:160-71.

3 Abdool Karim Q, Abdool Karim SS, Singh B, et al. Seroprevalence of HIV infection in rural South Africa. AIDS 1992;6:1535-9.

4 Colvin M, Abdool Karim SS, Wilkinson D. Migration and AIDS [letter]. Lancet 1995;346:1303-4.

5 Jochelson K, Mothibeli M, Leger J-P. Human immunodeficiency virus and migrant labour in South Africa. Int $\mathcal{F}$ Health Services 1991;21:157-73.

6 Kravitz JD, Mandel R, Petersen EA, et al. Human immunodeficiency virus seroprevalence in an occupational cohort in a South African community. Arch Intern Med 1995;155: $1601-4$.

7 Hudson CP. AIDS in rural Africa-a paradigm for prevention. Int F STD AIDS 1996;7:236-43.

8 Mohamed AO, Bwayo JJ, Mutere AN, et al. Sexual Mohamed AO, Bwayo J, Mutere AN, et al. Sexual
behaviour of long-distance truck drivers and their contribehaviour of long-distance truck drivers and their contri-
bution to the spread of sexually transmitted diseases and HIV infection in East Africa. VI International Conference on AIDS. San Francisco, June 1990 (abstract EC729).

9 Nzyuko S. Teenagers along the trans-African highway. AIDS Society 1991;2:10.

10 Tarantola D, Schwartlander B. HIV/AIDS epidemics in sub-Saharan Africa: dynamism, diversity and discrete declines? AIDS 1997;11 (suppl B):S5-21.

11 Grosskurth H, Mosha F, Todd I, et al. A community trial of the impact of improved sexually transmitted disease the impact of improved sexually transmitted disease line survey results. AIDS 1995;9:927-34.

12 Caraël M, Sexual behaviour. In: Cleland J, Ferry B, eds. Sexual behaviour and AIDS in the developing world. London: Taylor and Francis, 1995. 